\title{
Networked electric drives in the Industry 4.0
}

\author{
Giovanni Zanuso \\ Department of Electric Power and Energy Systems \\ KTH Royal Institute of Technology \\ Stockholm, Sweden \\ zanuso@kth.se \\ Luca Peretti \\ Department of Electric Power and Energy Systems \\ KTH Royal Institute of Technology \\ Stockholm, Sweden
}

\author{
Viktoria Fodor \\ Department for Network and Systems Engineering \\ KTH Royal Institute of Technology \\ Stockholm, Sweden
}

Oskar Wallmark

Department of Electric Power and Energy Systems

KTH Royal Institute of Technology

Stockholm, Sweden

\begin{abstract}
Industrial automation has been recently challenged by new initiatives such as Industry 4.0, which promises higher connectivity between the devices in an industrial plant. The goal of this work is to discuss how electric drives, widely employed in industry, could benefit from this increased connectivity. Specific applications, such as condition monitoring and multidrive systems, are considered to show the advantages of the industrial network presence, combined with the introduction of new data driven methods. Moreover, the status of industrial communication technologies is depicted, and their suitability for condition monitoring and multi-drive systems applications is described.
\end{abstract}

Index Terms - condition monitoring, electric drives, industrial networks, multi-drive systems

\section{INTRODUCTION}

In recent years, the introduction of methods and devices from the information and communication technology (ICT) world has enabled proposals on how to profoundly change the industry. In particular, the application of the Internet of Things and cyberphysical system concepts to the industrial context originated the "Industry 4.0" and the "Industrial Internet" initiatives in Germany and United States, respectively [1], [2]. The aims of these visions are a higher efficiency and flexibility, through an increased connectivity between the devices in an industrial plant and through the processing of information in cloud-based applications.

A fundamental factor in industrial automation is the electromechanical conversion, where electric drives, consisting of an electric motor supplied by a frequency converter, play a major role. Particularly during the last three decades, the need of performance and efficiency optimization, together with the necessity of process flexibility, have required an increased use of electric drives.

In current industrial plants electric drives are part of the two lowest hierarchical layers, known as the plant and the direct process control levels. The role of electric drives is limited to execute command references (e.g. position, speed

This work has been supported in part by Vinnova "Smartare elektroniksystem" strategic innovation program and the Swedish Electromobility Center (SEC). and torque) sent by the higher hierarchical layer, i.e. the supervisory level often implemented by a Programmable Logic Controller (PLC), and to respond with alarm conditions when faults occur [3]. This situation is rapidly changing due to innovative trends that increase the electric drive capabilities within the industrial context. The aim of this work is to discuss these trends.

Particular focus is put on the condition monitoring (CM) of the electric drive. Various methods for monitoring the status of both the electric motor components and the converter parts have been proposed [4]. Some of these methods exploit the presence of the processor in the converter, which is originally needed for performing the calculations required to supply the motor with suitable voltage references by means of pulsewidth modulation (PWM) strategies.

With the availability of a continuously increasing computational power, the converter processors can accomplish additional tasks, by exploiting all the information received from the sensors that are anyway installed in the electric drive. Given the amount of information that the converter can acquire and process, the electric drive could even extend its role. It could process information and make decisions locally, or directly exchange information with other drives participating in the same industrial plant in order to maximize the overall plant performance [5]. Moreover, direct access to the cloud infrastructure would allow data-analytics-based maintenance and product development.

All these possibilities rely massively on the communication capabilities of the electric drive with the other entities of the network. Therefore, in this work an overview of typical networks used for industrial communication is presented (fieldbuses and ethernet-based networks), as well as emerging technologies (wireless networks). A particular attention is put on the capabilities required to support the extended drive functionalities, such as the timely transmission of larger amount of data and traffic prioritization. It is then discussed which of these communication networks could support the new roles of the electric drives, and how processing and communication could be optimized, considering the processing power of the 
converter, the availability of a cloud infrastructure and the networking capabilities.

This paper is organized in the following parts. In Section II $\mathrm{CM}$ algorithms for electric drives are investigated. In Section III, multi-drive applications where the plant performance and efficiency can benefit from an increased communication capability of the electric drives are described. In Section IV the major types of communication technologies and their characteristics are explored. The drive requirements for some specific applications, in terms of data transmissions, are discussed in Section V, considering what current and emerging technologies can offer. Section VI reports final remarks about the work.

\section{Condition Monitoring Techniques for Electric DRIVES}

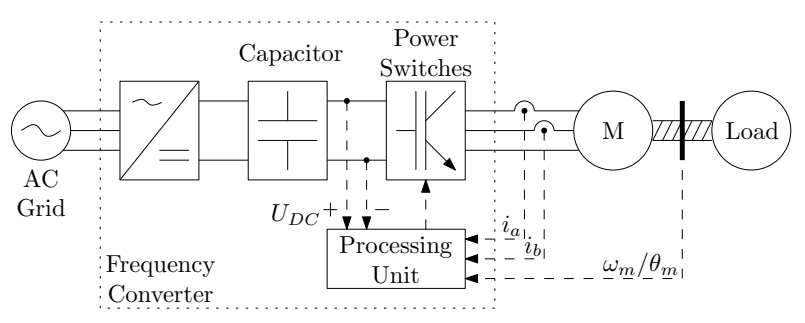

Fig. 1. Schematic overview of an electric drive.

Fig. 1 shows the schematic of a typical electric drive. The load represents the industrial plant part (e.g. fan, pump, actuator) that is put into motion by the electric motor. The frequency converter, on the other side, feeds the electric motor with pulse-width-modulated voltage in order to provide the required torque and speed. The only intelligence available in the whole drive system lies in the processing unit that controls the frequency converter. Its main function is to calculate and send the gate signals to the power switches (commonly insulated-gate bipolar transistor or metal-oxide-semiconductor field-effect transistors, namely IGBTs and MOSFETs respectively) to implement the PWM. In order to perform this task, the processing unit acquires, by means of sensors, inputs signals as two stator phase currents $\left(i_{a}, i_{b}\right)$, the DC-bus voltage $\left(U_{D C}\right)$ and, when sensorless algorithms are not implemented, the rotor speed $\left(\omega_{m}\right)$ or position $\left(\theta_{m}\right)$. It is worth noting that, in some electric drives, all three phase currents are measured. Additionally, signals monitoring the temperature of the motor and the frequency converter may also be present.

Together with cost, performance and efficiency, reliability is a key factor for the electric drives used in industrial plants. Failures in electric drives may lead to the whole plant shutting down with negative economic consequences. Unfortunately, electric drives consists of many electrical and mechanical components that are subject to failures. For example, in frequency converters the components that are more inclined to fail are the power switches and the DC-bus capacitors [6]. Failures occur also in the electric motor, both in its electrical components (stator windings, rotor bars) and its mechanical ones (bearings, rotor eccentricities).
Faults in electric drives can be early detected and possibly prevented by performing CM techniques combined with predictive maintenance procedures. In [4] the state of the art of $\mathrm{CM}$ for drive systems is described, whereas in [7] the most recent diagnostics techniques are highlighted. A general classification of CM methods for electric drives is based on whether external apparatuses or non-conventional measurements are required. Certainly, from a customer perspective, the preferable methods are those relying only on the sensors that are already present in the electric drives for control purposes. In this case, the additional costs of the CM techniques would depend only on the presence of additional software in the processing unit. A second general classification of CM methods is between online and offline techniques, where the former ones are favored because they perform during the electric drive normal operation, without the need to wait for it to be halted.

Traditional online CM techniques are based on motor current signature analysis (MCSA), where anomalies in the frequency spectrum of the measured stator currents are related to specific faults [8]. Nevertheless, electric drives are affected by many type of faults and distinguishing their effects in the current signature is a challenging task. Moreover, the application of MCSA in electric drives is more problematic than in AC-grid-connected motors because the current regulation presence makes the stator currents closer to sinusoidal signals, and thus masking the fault-related harmonics [9].

CM methods alternative to MCSA, and thus without its limitations, have been developed over the years. In [10], turnto-turn stator winding faults are detected by analyzing the effects of a high-frequency voltage injection. A completely different approach for the same fault type is used in [11], where the high-frequency oscillations of the stator currents, after a commutation of the power switches, are monitored. Such oscillations depend on the parasitic capacitances between turns, which values are related to the winding insulation properties. Thus, the insulation status can be monitored by analyzing the characteristics of the current oscillations. The described high-frequency ringing phenomenon is of particular interest for CM techniques because it is generally present in all kind of electrical drives.

Fig. 2 shows an example for a permanent-magnet synchronous machine (PMSM) used in commercial heavy hybrid vehicles. It is worth observing that the entire current ringing is a relatively fast phenomenon lasting for few $\mu s$. Therefore, the online implementation of such method in electric drives requires a high-frequency current sampling, also known as oversampling, obtainable with high-bandwidth current sensors and fast analog-to-digital converters (ADCs). This does not require additional equipment, but may need sensors and ADCs with increased capabilities.

Electric drives with oversampling capabilities may be beneficial for the development of other CM techniques. For example, in [12] aged and healthy power switches are distinguished by means of online measurements of their turn-off time during switching cycles, which can be possible with high-frequency sampling. 


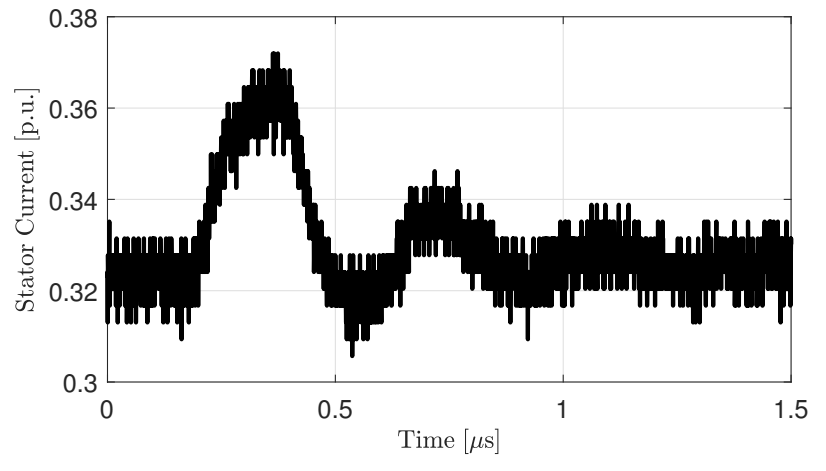

Fig. 2. Stator current ringing phenomenon measured in a PMSM.

The effectiveness of CM methods, both the traditional ones and those based on oversampled measurements, is often based on a comparison between current measurements and an initial reference measurement performed when the electric drive was supposedly healthy [4]. Moreover, diagnostics methods that take into account the whole history of measurements may be developed. The emergence of faults in electric drives is however a relatively slow process, which might take years if not decades. Therefore, in order to apply future's diagnostics techniques, large amounts of data need to be acquired and processed, which might be a matter of concern for the processing unit of the electric drive. Possible solutions are analyzed in Section V.

\section{MULTI-DRIVE SYSTEMS}

There are several industrial applications that can benefit from an increased communication between electric drives. Industrial plants involving multi-drive systems (e.g. conveyor belts, tandem-connected motors, robots) are natural examples. Fig. 3 shows a general diagram of a multi-drive system, where a number $n$ of electric drives share the same DC bus. Each frequency converter $\mathrm{FC}_{i}$ is equivalent to the one displayed in Fig. 1 without the presence of the AC/DC stage. The common DC bus gives the advantages of a reduced number of AC/DC stages (only one in Fig. 3) and the possibility to minimize the power flow from the $\mathrm{AC}$ grid, because the drives in motoring mode and those concurrently in generating mode can share the power on the DC side [13]. The energy excess present in the $\mathrm{DC}$ bus can be redirected to the $\mathrm{AC}$ grid, when a regenerative $\mathrm{AC} / \mathrm{DC}$ converter is employed, or be dissipated by balancing resistors.

Conventional conveyor belts are driven by multiple motors as in Fig. 3. Typically, in such systems induction motors are employed and the involved power may reach the order of MWs, making energy efficiency a critical factor. When the conveyor belt is not fully loaded, the system efficiency decreases because the losses in induction machines are higher at partial loads [5]. For this reason, a cooperative dynamic load sharing strategy, based on an increased exchange of information within the multi-drive system may decrease the total losses, compared to independent methods implemented in the drives.

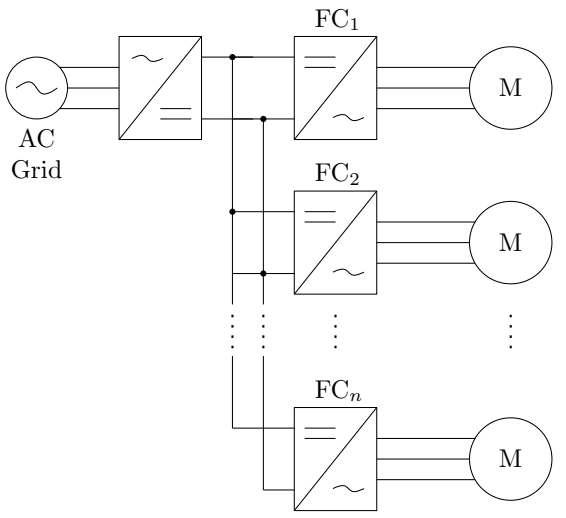

Fig. 3. General diagram of a multi-drive system.

It is worth noting that load sharing techniques are needed even for motors with equal power rating that run in the same operating point. In this case, there could still be a load unbalance caused by differences in motor parameters, which are temperature dependent [14]. Furthermore, power unbalances may be induced by the belt itself, especially during the starting process [15].

Load sharing and total loss minimization techniques are beneficial for other industrial applications where multi-drive systems are employed, for example when several motors are connected to the same load shaft, in the so-called tandemconnected configuration [16]. Such a multi-drive system with dynamic load sharing strategy is discussed in [17] for three synchronous motors connected to a centrifugal pump.

Differently from the conveyor belt systems and the tandemconnected motors, in industrial robot applications the motors of Fig. 3 are coupled to non-connected axes, and thus to independent loads. In this context, load sharing techniques are not applicable. However, the energy exchange in the DC bus between the different electric drives is critical for the total energy consumption [18]. When an axis decelerates, the mechanical energy is converted into electric energy implying a DC-bus voltage increase. With an abrupt axis braking or when the multiple axes decelerate at the same time, the DC-bus voltage level may increase excessively and thus the balancing resistor intervenes to dissipate the extra energy. Therefore, the system energy efficiency is negatively affected. This situation can be prevented if one or more axes accelerate during the DC-bus voltage increase. Such energy optimization problem can be tackled with strategies considering the robotic system as a whole, and thus requiring increased collaboration between its components. Similar issues are also present in other applications as port cranes, where multi-drive systems with independent loads are installed [19].

Multi-drive systems thus could benefit from the increased communication and collaboration. How this communication can be supported by industrial communication technologies is discussed in Section V. 


\section{Status OF Industrial COMMUNiCATION TECHNOLOGIES}

During the last three decades, the field of industrial communication has continuously evolved [20]. A large variation of communication protocols is now available for the electric drives in order to share information within an industrial network [21]. Industrial control requires hard real-time operations, implying that missing a deadline causes a total system failure. Under reasonable network loads, the hard real-time requirement demands synchronous access, meaning that the network nodes have the rights to access the transmission medium periodically. The typical access methods are polling, when a central controller sends to or requests information from the controlled nodes (the drives) in a round-robin manner, token passing, when transmission rights are forwarded through a virtual ring, and TDMA, with predefined transmission schedule. In addition, the remaining time can be accessible for non-real-time communication, often through random channel access with carrier sensing (CSMA).

The most traditional category is represented by the so-called fieldbus systems. Table I displays a few examples together with some characteristics, as the supported service, the mode in which the transmission medium is accessed (Channel Access) and the maximum obtainable transmission rate (Data Rate). Some of these solutions, like Profibus and CAN, provide only asynchronous access, and thus do not directly ensure the timely delivery of hard real-time messages. This instead have to be handled by proper network dimensioning [22]. Others, like ControlNet, can serve a mix of real-time and non-realtime traffic, providing both synchronous and asynchronous service. This is typically achieved by the introduction of a slotted time structure, where a part of a time-slot is reserved for synchronous, and and the other part for asynchronous transmissions.

It is worth noting that the maximum data rate values given for the various communication protocols listed in this section are simple approximations because the actual values depend on several network characteristics, like the network diameter and the node hardware.

TABLE I

SELECTED INDUSTRIAL FIELDBUS PROTOCOLS.

\begin{tabular}{|c|c|c|c|}
\hline Protocol & Service & Channel Access & Data Rate \\
\hline Profibus DP & Async & Polling [20] & $12 \mathrm{Mbps}$ \\
CAN & Async & CSMA+prio [22] & $1 \mathrm{Mbps}$ \\
ControlNet & Sync + Async & Token Passing [20] & $5 \mathrm{Mbps}$ \\
\hline
\end{tabular}

The second category of industrial communication protocols is based on the Ethernet technology, but extended with realtime capabilities [21], [23]. Table II lists some examples of the real-time Ethernet protocols. These solutions clearly guarantee a higher transmission rate with respect to the fieldbus systems, and the need for providing both synchronous and asynchronous access is widely recognized.

The third and most recently developed category of industrial communication protocols is based on wireless communica-
TABLE II

SELECTED REAL-TIME ETHERNET PROTOCOLS.

\begin{tabular}{|c|c|c|c|}
\hline Protocol & Service & Channel Access & Data Rate \\
\hline PROFINET & Sync + Async & Polling & $100 \mathrm{Mbps}$ \\
EtherCat & Sync + Async & Polling & $100 \mathrm{Mbps}$ \\
Ethernet Powerlink & Sync + Async & Polling & $100 \mathrm{Mbps}$ \\
\hline
\end{tabular}

tions. Wireless communication, on one hand can ensure the increased flexibility of the plants, on the other hand, the communication itself may be unreliable in the harsh industrial environment. Table III displays a selection of industrial wireless protocols [24]. As a consequence of the different transmission medium, the maximum data rates are considerably lower than for the fieldbus and real-time Ethernet protocols. Moreover, as these systems use open spectrum bands, the transmissions may be disturbed by other wireless networks transmitting in the same area.

TABLE III

SELECTED INDUSTRIAL WIRELESS PROTOCOLS.

\begin{tabular}{|c|c|c|c|}
\hline Protocol & Service & Channel Access & Data Rate \\
\hline Wireless Hart & Sync & TDMA & $250 \mathrm{kbps}$ \\
ISA100.11a & Sync & TDMA & $250 \mathrm{kbps}$ \\
Zigbee & Sync + Async & TDMA+CSMA & $250 \mathrm{kbps}$ \\
6LoWPAN & Sync + Async & Polling+CSMA & $250 \mathrm{kbps}$ \\
\hline
\end{tabular}

In addition to these dedicated wireless industrial sensor network technologies, modern mobile telecommunication systems could be good candidates to support industrial communication [1]. The advantage of LTE and the emerging 5G networks is twofold. First, the use of dedicated spectrum can guarantee high data rates and strict transmission deadlines. Second, these systems can serve a diversity of applications, which allows enterprises to use one single networking technology on all layers. The advantages come of course with the drawbacks of complex and costly communication hardware and the additional cost of spectrum lease [25].

\section{ApPliCATIONS AND ANALYSiS}

In this section three industrial application examples described in Section II and Section III are analyzed from a data communication perspective. For the investigated cases, the suitability of the communication technologies reported in Section IV is discussed.

\section{A. Condition monitoring with MCSA or similar methods}

Traditional CM methods as the MCSA require the acquisition of signals having the characteristics displayed in Table IV. The listed values are not meant to be accurate in absolute terms, but they can be considered as a possible case study.

Generally, MCSA techniques acquire signals at the same PWM frequency (e.g. $f_{c}=10 \mathrm{kHz}$ ) with a specific observation time window $T_{\text {obs }}$. The latter parameter is fundamental because it determines the frequency resolution $d f$ of the resulting frequency spectrum, being $d f=1 / T_{\text {obs }}$ when a Discrete Fourier Transform (DFT) is applied. The resulting $d f=0.2 \mathrm{~Hz}$ 
TABLE IV

SIGNALS CHARACTERISTICS FOR CM WITH MCSA.

\begin{tabular}{|c|c|c|}
\hline Parameter & Symbol & Value \\
\hline Current signals amount & $N_{c}$ & 3 \\
Speed/position signals amount & $N_{s}$ & 1 \\
Signals resolution & $b$ & $12 \mathrm{bit}$ \\
Sampling frequency & $f_{c}$ & $10 \mathrm{kHz}$ \\
Observation time window & $T_{o b s}$ & $5 \mathrm{~s}$ \\
Number of measurements & $N_{m}$ & 10 \\
\hline
\end{tabular}

is reasonable with respect to common MCSA methods [8]. Moreover, it is assumed that CM methods will be applied to a consecutive number of measurements $N_{\text {meas }}$ for a greater accuracy.

The data size originated by one single measurement session is:

$$
P_{C M}=\frac{\left(N_{c}+N_{s}\right) b f_{c} T_{o b s} N_{m}}{8}=3 \text { MBytes. }
$$

The measurement sessions are repeated periodically with a very low frequency (hours or even days) given the slowness of the degrading phenomena in electric drives. Between consecutive measurement sessions, the obtained amount of data $P_{C M}$ needs to be adequately processed, according to the specific $\mathrm{CM}$ technique. The involved data size and the type of processing required (DFT) might exceed the computational limitation of the electric drive's processing unit, which in the meanwhile needs to perform continuously control-related tasks within stringent time constraints.

An alternative is the communication of the acquired data to an external processing unit, which can in turn perform $\mathrm{CM}$ algorithms and store the results for historical-data-based diagnostics. A possibility is to perform the mentioned processing in the cloud, connected to all the industrial plant's electric drives.

The transmission of the $\mathrm{CM}$ data $P_{C M}$ does not have realtime constraints, and should be transmitted through asynchronous access in the industrial network, in the time that is left by the more demanding control applications. Most of the technologies in Tables I-III would support this communication and provide data delivery within minutes.

\section{B. Condition monitoring with oversampling-based methods}

Innovative CM methods based on oversampling, as the one described in [11], need to acquire signals with the characteristics displayed in Table V.

TABLE V

SIGNALS CHARACTERISTICS FOR OVERSAMPLING-BASED CM.

\begin{tabular}{|c|c|c|}
\hline Parameter & Symbol & Value \\
\hline Current signals amount & $N_{c}$ & 3 \\
Signals resolution & $b$ & $12 \mathrm{bit}$ \\
Oversampling frequency & $f_{o s}$ & $50 \mathrm{MHz}$ \\
Observation time window & $T_{o b s}$ & $10 \mu \mathrm{s}$ \\
Number of measurements & $N_{m}$ & 10 \\
\hline
\end{tabular}

Considerations done in Section V-A apply also to this case. The chosen $T_{o b s}$ value, comparable to the one of [11] and with the measurement of Fig. 2, gives a frequency resolution $d f=$ $100 \mathrm{kHz}$ that is reasonable because the ringing phenomenon is in the order of $\mathrm{MHz}$.

The data size originated by one single measurement session is:

$$
P_{O S}=\frac{N_{c} b f_{o s} T_{o b s} N_{m}}{8}=22.5 \mathrm{kBytes}
$$

that is two orders of magnitude lower than the case studied in Section V-A. Therefore, in this case, the local processing unit may be more suitable to perform CM techniques. Whether the data need to be sent to an external processing unit (or to a cloud), the same considerations done in Section V-A apply, but with more relaxed constraints.

\section{Centralized control in a multi-drive system}

As stated in Section III, in multi-drives systems, loadsharing strategies, total losses minimization techniques and DC-bus energy optimization methods will benefit from a collaborative network of electric drives. A particularly interesting situation is when a centralized control takes completely care of the reference generation for the single electric drives in the system. In this manner, the centralized control will have all the data available coming from each of the electric drives in order to perform the required optimization techniques. However, it is assumed that all the local processing units still performs low-level tasks such as communication with drive sensors and PWM. This may potentially induce a reduction of the computational power required for the local processing units and therefore impact the system cost.

The signals exchanged between the centralized control unit and each single electric drive in the network have the characteristics displayed in Table VI. Differently from what reported

TABLE VI

SIGNALS CHARACTERISTICS FOR A CENTRALIZED CONTROL IN A MULTI-DRIVE SYSTEM.

\begin{tabular}{|c|c|c|}
\hline Parameter & Symbol & Value \\
\hline Current signals amount & $N_{c}$ & 2 \\
DC-bus voltage signals amount & $N_{v}$ & 1 \\
Speed/position signals amount & $N_{s}$ & 1 \\
PWM reference signals amount & $N_{r e f}$ & 3 \\
Signals resolution & $b$ & $12 \mathrm{bit}$ \\
Sampling frequency & $f_{c}$ & $10 \mathrm{kHz}$ \\
\hline
\end{tabular}

in Section V-A and Section V-B, the data communication must respect strict time constraints. At each PWM period, equal to the sampling period $T_{c}=1 / f_{c}$, the measurements of currents, DC-bus voltage and speed (or position) are transmitted to the central processing unit, which calculates the references to be sent back to the local PWM modulator of each electric drive.

The data rate of the exchange between the central processing unit and one single local drive is:

$$
R_{M D}=\left(N_{c}+N_{v}+N_{s}+N_{r e f}\right) b f_{c}=840 \mathrm{kbps} .
$$

The obtained result should be considered only for its order of magnitude, because overhead bits required in the transmission are not take into account. 
The total network transmission rate should consider the transmissions from all the electric drives in the system, with the small modification that $N_{v}$ needs to be transmitted only once, because the DC-bus voltage value is the same for all drives.

All in all, the transmission rate requirement of the multidrive system is high compared to the requirement of traditional drive control. The wired real-time Ethernet solutions in Table II can support this application. In the wireless domain multi-drive control can provide a good use-case for LTE and $5 \mathrm{G}$ systems, since the required data rates are not supported by the industrial wireless sensor network standards.

\section{CONCLUSIONS}

In this work, trends regarding the use of electric drives in the Industry 4.0 were described and analyzed. Specific applications as condition monitoring and multi-drive systems were put into focus. In these applications, the connection of electric drives to a communication network was shown to be beneficial. Particularly, possibilities of external processing of data for condition monitoring and centralized control in multidrives systems were discussed.

Considering communication requirements, modern wired industrial network technologies can well support condition monitoring and multi-drive systems. However, the wireless solutions need to provide higher transmission rates to support centralized real-time multi-drive control.

\section{REFERENCES}

[1] M. Wollschlaeger, T. Sauter, and J. Jasperneite, "The Future of Industrial Communication: Automation Networks in the Era of the Internet of Things and Industry 4.0," IEEE Ind. Electron. Mag., vol. 11, no. 1, pp. 17-27, Mar. 2017.

[2] A. W. Colombo, S. Karnouskos, O. Kaynak, Y. Shi, and S. Yin, "Industrial Cyberphysical Systems: A Backbone of the Fourth Industrial Revolution," IEEE Ind. Electron. Mag., vol. 11, no. 1, pp. 6-16, Mar. 2017.

[3] F. Benzi, G. S. Buja, and M. Felser, "Communication architectures for electrical drives," IEEE Trans. Ind. Informat., vol. 1, no. 1, pp. 47-53, Feb. 2005.

[4] M. Riera-Guasp, J. A. Antonino-Daviu, and G. A. Capolino, "Advances in Electrical Machine, Power Electronic, and Drive Condition Monitoring and Fault Detection: State of the Art," IEEE Trans. Ind. Electron., vol. 62, no. 3, pp. 1746-1759, Mar. 2015.

[5] M. P. Sruthi, C. Nagamani, and G. S. Ilango, "Dynamic Load Sharing in Multi-Machine Conveyor Belt Systems," in 2017 IEEE PES Asia-Pacific Power and Energy Engineering Conference (APPEEC), Nov. 2017, pp. $1-6$.

[6] S. Yang, A. Bryant, P. Mawby, D. Xiang, L. Ran, and P. Tavner, "An Industry-Based Survey of Reliability in Power Electronic Converters," IEEE Trans. Ind. Appl., vol. 47, no. 3, pp. 1441-1451, May 2011.

[7] G. A. Capolino, J. A. Antonino-Daviu, and M. Riera-Guasp, "Modern Diagnostics Techniques for Electrical Machines, Power Electronics, and Drives," IEEE Trans. Ind. Electron., vol. 62, no. 3, pp. 1738-1745, Mar. 2015.

[8] J. H. Jung, J. J. Lee, and B. H. Kwon, "Online Diagnosis of Induction Motors Using MCSA," IEEE Trans. Ind. Electron., vol. 53, no. 6, pp. 1842-1852, Dec. 2006

[9] A. Bellini, F. Filippetti, C. Tassoni, and G. A. Capolino, "Advances in Diagnostic Techniques for Induction Machines," IEEE Trans. Ind. Electron., vol. 55, no. 12, pp. 4109-4126, Dec. 2008.

[10] F. Briz, M. W. Degner, P. Garcia, and A. B. Diez, "High-Frequency Carrier-Signal Voltage Selection for Stator Winding Fault Diagnosis in Inverter-Fed AC Machines," IEEE Trans. Ind. Electron., vol. 55, no. 12, pp. 4181-4190, Dec. 2008.
[11] P. Nussbaumer, M. A. Vogelsberger, and T. M. Wolbank, "Induction Machine Insulation Health State Monitoring Based on Online Switching Transient Exploitation," IEEE Trans. Ind. Electron., vol. 62, no. 3, pp. 1835-1845, Mar. 2015.

[12] D. W. Brown, M. Abbas, A. Ginart, I. N. Ali, P. W. Kalgren, and G. J. Vachtsevanos, "Turn-Off Time as an Early Indicator of Insulated Gate Bipolar Transistor Latch-up,' IEEE Transactions on Power Electronics, vol. 27, no. 2, pp. 479-489, Feb. 2012.

[13] T. M. Parreiras, J. C. G. Justino, A. V. Rocha, and B. d. J. C. Filho, "True Unit Power Factor Active Front End for High-Capacity Belt-Conveyor Systems," IEEE Trans. Ind. Appl., vol. 52, no. 3, pp. 2737-2746, May 2016.

[14] J. Iyer, M. Chapariha, F. Therrien, and J. Jatskevich, "Improved torque sharing in multi induction motor VFD systems using current feedback," in $201225^{\text {th }}$ IEEE Canadian Conference on Electrical and Computer Engineering (CCECE), Apr. 2012, pp. 1-5.

[15] D. Xiao, X. Li, and K. He, "Power Balance of Starting Process for Pipe Belt Conveyor Based on Master-Slave Control," IEEE Access, vol. 6, pp. 16924-16931, Mar. 2018.

[16] A. Kurt, A. K. Atalay, and D. A. Kocabas, "Load sharing analysis of identical wound rotor induction motors driving the same load," in 2016 International Automatic Control Conference (CACS), Nov. 2016, pp. 247-251.

[17] M. Bernia and P. Sicard, "A hierarchical algorithm for load sharing in Multi-machine Multi-converter Systems with flexible coupling," in 2015 IEEE $28^{\text {th }}$ Canadian Conference on Electrical and Computer Engineering (CCECE), May 2015, pp. 1151-1155.

[18] D. Meike, M. Pellicciari, and G. Berselli, "Energy Efficient Use of Multirobot Production Lines in the Automotive Industry: Detailed System Modeling and Optimization," IEEE Trans. Autom. Sci. Eng., vol. 11, no. 3, pp. 798-809, Jul. 2014.

[19] G. Parise, L. Parise, A. Malerba, F. M. Pepe, A. Honorati, and P. B. Chavdarian, "Comprehensive Peak-Shaving Solutions for Port Cranes," IEEE Trans. Ind. Appl., vol. 53, no. 3, pp. 1799-1806, May 2017.

[20] T. Sauter, "The Three Generations of Field-Level Networks - Evolution and Compatibility Issues," IEEE Trans. Ind. Electron., vol. 57, no. 11, pp. 3585-3595, Nov. 2010 .

[21] B. Galloway and G. P. Hancke, "Introduction to Industrial Control Networks," IEEE Communications Surveys Tutorials, vol. 15, no. 2, pp. 860-880, 2013.

[22] R. I. Davis, A. Burns, R. J. Bril, and J. J. Lukkien, "Controller Area Network (CAN) schedulability analysis: Refuted, revisited and revised," Real-Time Systems, vol. 35, no. 3, pp. 239-272, 2007.

[23] S. Vitturi, L. Peretti, L. Seno, M. Zigliotto, and C. Zunino, "Realtime Ethernet networks for motion control," Computer Standards \& Interfaces, vol. 33, no. 5, pp. 465-476, Sep. 2011.

[24] M. Raza, N. Aslam, H. Le-Minh, S. Hussain, Y. Cao, and N. M. Khan, "A Critical Analysis of Research Potential, Challenges, and Future Directives in Industrial Wireless Sensor Networks," IEEE Communications Surveys Tutorials, vol. 20, no. 1, pp. 39-95, 2018.

[25] M. Gidlund, T. Lennvall, and J. Åkerberg, "Will 5G Become Yet Another Wireless Technology for Industrial Automation?" in 2017 IEEE International Conference on Industrial Technology (ICIT), Mar. 2017, pp. 1319-1324. 